\title{
COVID 19: Evaluate of Liver and Renal Function Tests in Iraqi Patients
}

\author{
Sura A. Abdulsattar ${ }^{1}$, Mahmood Nazar Mustafa ${ }^{2}$, Abdullah Ali Mohammed ${ }^{3}$, Ahmed Salam Al-zaidi ${ }^{4}$

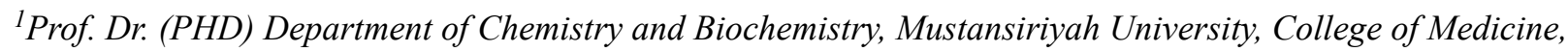 \\ Iraq, ${ }^{2}$ Assistant Lecturer (MSc) Department of Pathology and Forensic Medicine, University of Fallujah, College \\ of Medicine, Iraq, ${ }^{3}$ Assistant Lecturer (MSc) Department of Chemistry and Biochemistry, University of Fallujah, \\ College of Medicine, Iraq, ${ }^{4}$ (MSc) Department of Medical Analysis, Al-Furat General Hospital, Iraq
}

\begin{abstract}
The clinical features of COVID-19 are varied, ranging from asymptomatic state to acute respiratory distress syndrome and multi organ dysfunction. We aim to evaluate renal and liver functions of patients with COVID 19. Laboratory results were obtained from 107 patients with laboratory-confirmed COVID-19 whowere admitted to the only Al-Furat General Hospital in Baghdad, Iraq from March 3 to June 9, 2020 and followed up until recovery. Normal levels of renal functions were presented. Meanwhile elevated levels of alanine aminotransferase (ALT) was observed in $10 \%$ and of aspartate aminotransferase (AST) was observed in $40 \%$ of patients with COVID 19,yet on comparison of the results at entering with at recovery it was observed significant differences $(\mathrm{p}<0.01)$ of all patients. From these findings we conclude that the virus might be responsible for systemic inflammation.
\end{abstract}

Keyword: COVID 19; liver enzyme;urea; creatinine; AST, ALT.

\section{Introduction}

On 7 January 2020, a novel coronavirus was identified in the throat swab sample of one patient by the Chinese Center for Disease Control and Prevention (CDC), and was subsequently named as $2019 \mathrm{nCoV}$ by World Health Organization (WHO) ${ }^{1,2}$. The virus was identified as a coronavirus that had $>95 \%$ homology with the bat coronavirus and $>70 \%$ similarity with the SARS- CoV. Environmental samples from the Huanan sea food market also tested positive, signifying that the virus originated from there ${ }^{3}$. The number of cases started increasing exponentially, some of which did not have exposure to the live animal market, suggestive of the fact that human-to-human transmission was

\section{Corresponding Author:}

\section{Abdullah Ali Mohammed}

Assistant Lecturrer (MSc) Department of Chemistry and Biochemistry, University of Fallujah, College of Medicineabdullah

e-mail: medical@yahoo.com occurring $(2020)^{4}$. All ages are susceptible. Infection is transmitted through large droplets generated during coughing and sneezing by symptomatic patients but can also occur from asymptomatic people and before onset of symptoms ${ }^{5}$. The clinical features of COVID-19 are varied, ranging from asymptomatic state to acute respiratory distress syndrome and multi organ dysfunction. The common clinical features include fever (not in all), cough, sore throat, headache, fatigue, headache, myalgia and breathlessness ${ }^{6}$. Some patients with COVID-19 pneumonia also present with kidney injury, and autopsy findings of patients who died from the illness sometimes show renal damage. However, little is known about the clinical characteristics of kidney-related complications, including hematuria, proteinuria, and AKI ${ }^{7}$. Recently, there has been some insight into the impact of COVID-19 on other organs, as a number of reports have indicated that more than half of patients with COVID-19 showed varying levels of liver disease ${ }^{8}$. The median time from onset of symptoms to dyspnea was five day, hospitalization seven day and acute respiratory distress syndrome (ARDS) eight day. The need for intensive care admission was in 25-30\% of affected patients in published series. Complications 
witnessed included acute lung injuryacute cardiac injury, shock and acute kidney injury. Recovery started in the 2 nd or $3 \mathrm{rd} \mathrm{wk}^{9}$. The various diagnosis method such as serological, molecular, and radiological can help the health centers in the detection of SARS-CoV-2; radiological and serological techniques are the best method among the others and the radiological method is the most preferred one, able to diagnose the infection quickly and accurately with fewer false-negatives ${ }^{10}$. Liver impairment has been reported as a common clinical manifestation in patients with SARS-CoV infection, even if not a prominent feature of the illness ${ }^{11,12}$.

\section{Material and Method}

This was a cross-sectional study, we analyzed biochemical data from electronic medical records of 107 hospitalized patients of Al-Furat General Hospital from Baghdad with COVID-19 (65 male and 42 female) for period (march 3 to June 9,2020). The data analysis included urea, creatinine, ALT, AST, and ALP twice: first at entering the hospital (group A) and second at recovery (group B).

Statistical Analysis: Continuous data were expressed as mean and standard deviation (SD). For the variables, paired test was employed to analyse the difference. All statistical analyses were performed using the SPSS 20.0 (SPSS Inc) software package. A (P value of $<0.05$ ) was considered statistically significant.

\section{Results and Discussion}

The current evidence indicates that infection rates of COVID 19 are higher in male than in female (Figure 1), where $61 \%$ (65 among 107) was male and 39\% (42 among 107) was female.

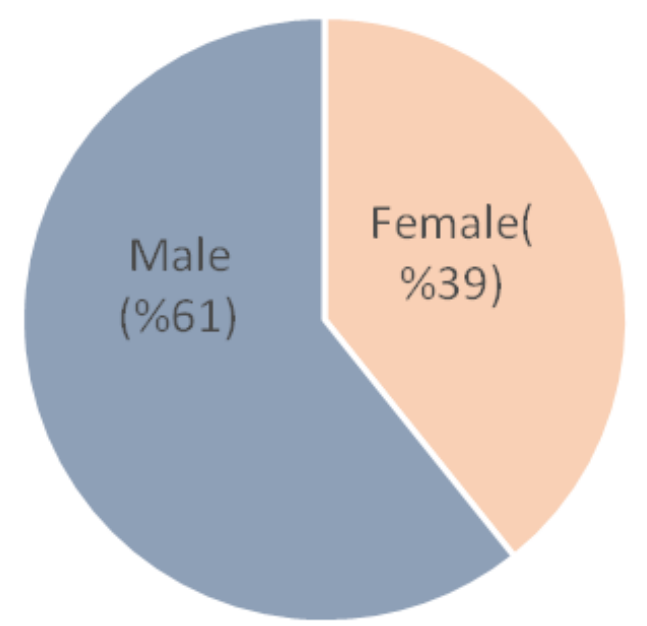

Figure 1: Covid 19 distribution among gender
The current results of COVID 19 distribution among age as shown in Figure 2 indicated that the most affected ages are between (20-40 year). The lowest affected ages was less than 20 year.

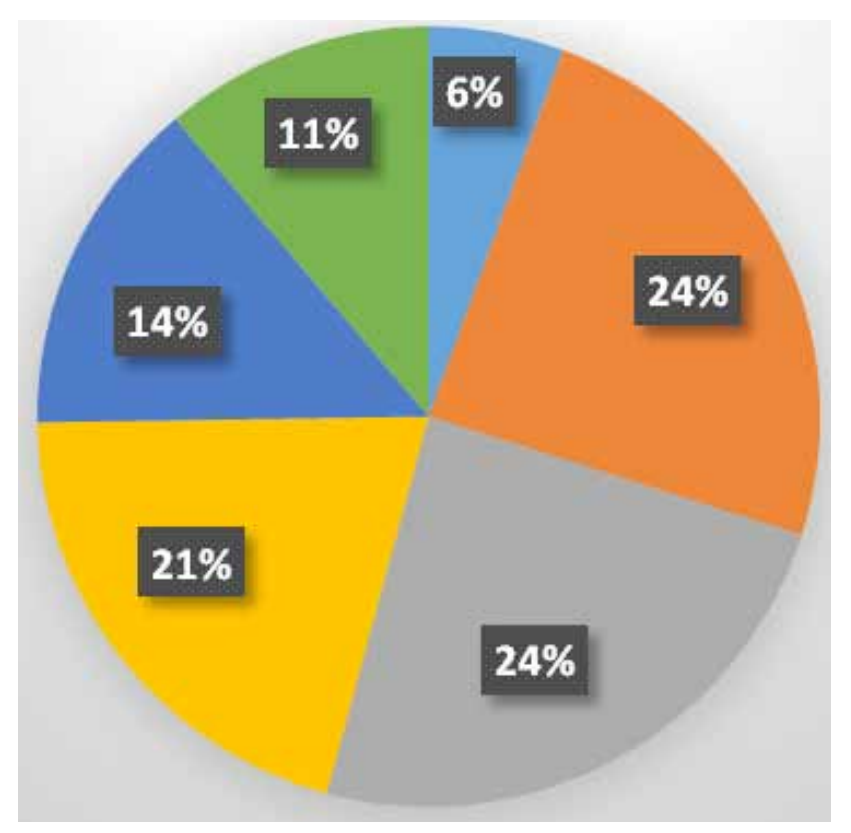

Figure 2: Covid 19 distribution among age where $6 \%$ : patients with age $<20$ year, $24 \%$ : patients with age range (21-30 year) \& (31-40 year)respectively, $21 \%$ : : patients with age range(41-50 year), $14 \%$ :: patients with age range(51-60year), and $11 \%$ : patients with age (61-70 year).

The results presented in Table 1 shows the levels of urea, creatinine, AST, ALT and ALP in sera of COVID 19 patients over two periods: the first when entering the hospital (group A) and the second when recovery (group B). Levels of urea, creatinine, AST, ALT and ALP were showed within normal level, however there were a highly significant differences $(\mathrm{p}<0.01)$ between groups in urea, creatinine, AST in both male and female patients .While the results of ALT indicated highly significant differences $(p<0.01)$ only in male patients. For ALP the results of male indicated highly significant differences $(p<0.01)$ and for female the result showed significant differences $(p<0.05)$. In more detail the results indicted elevated levels of AST in $40 \%$ patients and of ALT in $10 \%$ patients, while no elevated levels of ALP was observed. 
Table 1: The levels of urea, creatinine, AST, ALT and ALP in sera of COVID 19 patients

\begin{tabular}{|l|l|c|c|c|}
\hline Parameter & Gender & Mean \pm SD Group A & Mean \pm SD Group B & P value \\
\hline \multirow{2}{*}{ Urea $(\mathrm{mmol} / \mathrm{L})$} & Male & 4.29 & 3.48 & 0.00 \\
\cline { 2 - 5 } & Female & 4.33 & 3.21 & 0.00 \\
\hline \multirow{3}{*}{ Creatinine $(\mathrm{mmol} / \mathrm{L})$} & Male & 74.58 & 69.67 & 0.00 \\
\cline { 2 - 5 } & Female & 72.78 & 68.47 & 0.00 \\
\hline \multirow{3}{*}{ AST $(\mathrm{U} / \mathrm{L})$} & Male & 45.96 & 36.26 & 0.00 \\
\cline { 2 - 6 } & Female & 45.7857 & 36.1429 & 0.00 \\
\hline \multirow{2}{*}{ ALP $(\mu \mathrm{kat} / \mathrm{L})$} & Male & 36.41 & 28.83 & 0.00 \\
\cline { 2 - 6 } & Female & 26.2381 & 24.2143 & 0.106 \\
\cline { 2 - 6 } & Male & 81.63 & 76.10 & 0.00 \\
\hline
\end{tabular}

Although available sex-disaggregated data for COVID-19 show equal numbers of cases between sexes, current study indicates that infectious rates are higher in male than in female, and that may be due to sex hormone that contribute to different immunologic responses in men and women: As a general rule, estrogens promote both innate and adaptive immune responses, which result in faster clearance of pathogens and greater vaccine efficacy ${ }^{13}$. Conversely, testosterone has largely suppressive effects on immune function, which may explain the greater susceptibility to infectious diseases observed in men ${ }^{14}$. Notably, changes in sex hormone may further shape the immune response to pathogens ${ }^{14}$. Sex-related biological data may also be critical to investigate the contribution of sex hormones in inflammatory response. In particular, reduction in testosterone levels in aging men has been associated with increased proinflammatory cytokine levels ${ }^{14}$ which may contribute to worse COVID-19 progression in older men. Sex differences in disease progression may also be linked to estrogen-induced decreased expression of angiotensin-converting enzyme $2^{15}$ Pre-print studies are conflicting as to whether ACE2 expression in lung tissue is different between sexes ${ }^{16,17}$. Some patients with COVID-19 pneumonia also present with kidney injury, and autopsy findings of patients who died from the illness sometimes show renal damage ${ }^{7}$. In meta-analysis study of Zhu $\mathrm{j}$ et al indicated that some patients with COVID 19 (25.5\%) presented elevated levels of renal functions ${ }^{18}$. The results of current study indicated that all patients presented normal levels of renal functions. However on comparison of the results at entering with at recovery it was observed significant differences $(\mathrm{p}<0.01)$. Patients with abnormal liver tests were at increased risk of progressing to severe disease. The detrimental effects on liverinjury mainly related to certain medications used during hospitalization,and should be monitored and evaluated frequently ${ }^{18}$.

\section{Conclusion}

Elevated levels of alanine aminotransferase (ALT) was observed in $10 \%$ and of aspartate aminotransferase (AST) was observed in $40 \%$ of patients with COVID 19 ,yet on comparison of the results at entering with at recovery it was observed significant differences $(p<0.01)$ of all patients. As AST is not specific for liver damage, which indicated that the systemic inflammation induced by the virus might be responsible for these findings not related to certain medication.

Conflict of Interest Statement: All authors declare that they have no conflict of interest.

\section{Source of Funding: Self}

Ethical Clearance: Taken from Al-Furat general hospital-Iraq.

\section{References}

1. Huang C, Wang Y, Li X, Ren L, Zhao J, Hu Y, Zhang L, Fan G, Xu J, Gu X, Cheng Z, Yu T, Xia J, Wei Y, Wu W, Xie X, Yin W, Li H, Liu M, Xiao Y, Gao H, Guo L, Xie J, Wang G, Jiang R, Gao Z, Jin Q, Wang J, Cao B. Clinical features of patients infected with 2019 novel coronavirus in Wuhan, China. Lancet. 2020; 395:497-506.

2. Chen N, Zhou M, Dong X, Qu J, Gong F, Han $\mathrm{Y}$, et al. (2019), Epidemiological and clinical 
characteristics of 99 cases of novel coronavirus pneumonia in Wuhan, China: a descriptive study. Lancet. 2020;395:507-513.

3. Xinhua. China's CDC detects a large number of new coronaviruses in the South China seafood market in Wuhan.2020; Available at: https://www. xinhuanet.com/2020-01/27/c_1125504355.htm.

4. Liu J, Shu YL, and Zhou XN. Transmission patterns and control of COVID-19 epidemic.Indian Journal of plant physiology .2020; 9:112.

5. Rothe Camilla, Schunk Mirjam, Sothmann Peter, Bretzel Gisela, Froeschl Guenter, Wallrauch Claudia, Zimmer Thorbjörn, Thiel Verena, Janke Christian, Guggemos Wolfgang, Seilmaier Michael, Drosten Christian, Vollmar Patrick, Zwirglmaier Katrin, Zange Sabine, Wölfel Roman, Hoelscher Michael. Transmission of 2019-nCoV Infection from an Asymptomatic Contact in Germany. New England Journal of Medicine. 2020;(10):970-971.

6. Singhal T..A Review of Coronavirus Disease-2019 (COVID-19).Indian J Pediatr.2020; 87(4): 281286.

7. Guangchang Pei' Zhiguo Zhang, Jing Peng' Liu Liu' Chunxiu Zhang, Chong Yu' Zufu Ma' Yi Huang, Wei Liu' Ying Yao' Rui Zeng, Gang Xu . (2020 Apr 28), Renal Involvement and Early Prognosis in Patients with COVID-19 Pneumonia.J Am Soc Nephrol. 2020; 31(6):1157-1165.

8. Cai Q, Huang D, Yu H, Zhu Z, Xia Z, Su Y, Li Z, Zhou G, Gou J, Qu J, SunY, Liu Y, He Q, Chen J, Liu L, Xu L, Characteristics of Liver Tests in COVID-19 Patients, Journal ofHepatology .2020;April.

9. Chau TN, Lee KC, Yao H, Tsang TY, Chow TC, Yeung YC, et al. . SARS-associated viral hepatitis caused by a novel coronavirus: report of three cases. Hepatology.2004; 39:302-310.
10. Ozma MA, Maroufi P, Khodadadi E, Köse S, Esposito I, Ganbarov K, Dao S, Esposito S, Dal T, Zeinalzadeh E, Kafil HS. Clinical manifestation, diagnosis, prevention and control of SARS-CoV-2 (COVID-19) during the outbreak period.Infez Med. 2020;28(2):153-165.

11. Humar A, McGilvray I, Phillips MJ, Levy GA. Severe acute respiratory syndrome and the liver. Hepatology.2004; 39: 291- 294.

12. Chan HLY, Leung W-K, To K-F, et al. Retrospective analysis of liver function derangement in severe acute respiratory syndrome. Am J Med.2004; 116: 566- 567.

13. Bartz D, Chitnis T, Kaiser UB, et al. (2020), Clinical advances in sex- and gender-informed medicine to improve the health of all: a review. JAMA Intern Med. [PMID: 32040165] doi:10.1001/ jamainternmed.2019.7194GOOGLE SCHOLAR.

14. Bianchi VE. . The anti-inflammatory effects of testosterone. J Endocr Soc.2019;3:91-107.

15. La Vignera S, Cannarella R, Condorelli RA, et al. Sex-specific SARS-CoV-2 mortality: among hormone-modulated ACE2 expression, risk of venous thromboembolism and hypovitaminosis $\mathrm{d}$. Int J Mol Sci.2020;21.

16. Cai G. Bulk and single-cell transcriptomics identify tobacco-use disparity in lung gene expression of ACE2, the receptor of 2019-nCov. MedRxiv, published online .2020; February 02.

17. Zhao Y, Zhao Z, Wang Y, Zhou Y, Ma Y, Zuo W. Single-cell RNA expression profiling of ACE2, the putative receptor of Wuhan 2019-nCov. BioRxiv, 2020; 26

18. Jieyun Zhu, Pan Ji, Jielong Pang, Zhimei Zhong, Hongyuan $\mathrm{Li}$, Cuiying $\mathrm{He}$, Jianfeng Zhang, Chunling Zhao. Clinical Characteristics of 3062 COVID-19 Patients: A Meta-Analysis.J Med Virol. 2020; Apr 15 : 10.1002/jmv.25884. 